\title{
Definición de un modelo para la planeación financiera personal aplicado al caso colombiano*
}

\author{
Miguel David Rojas López** \\ Lina Marcela Maya Fernández $z^{* * *}$ \\ María Elena Valencia ${ }^{* * * *}$
}

Recibido: 09/10/2015 • Aceptado: 15/11/2016

DOl: 10.22395/rium.v16n31a8

\begin{abstract}
Resumen
Planeación financiera personal es un conjunto de actividades requeridas para definir objetivos, establecer de planes de acción y satisfacer necesidades financieras de individuos. Diferentes autores representan el proceso de planeación financiera personal a través de modelos presentando desventajas para aplicarlos en el ámbito colombiano porque han sido creados en países desarrollados, suponen la existencia de una industria de planeación financiera y no existen estudios académicos que los adapten para las necesidades propias del entorno. Para solucionar estas desventajas se propone en esta investigación la definición de un modelo unificador de planeación financiera personal, que agrupe los diferentes elementos que se identifican a partir de los modelos estudiados y que tenga aplicación en el entorno colombiano.
\end{abstract}

Palabras clave: Finanzas personales, planeación financiera personal, educación financiera.

* El artículo se deriva de una investigación que soporta una tesis de maestría en ingeniería administrativa durante 2015. El artículo se trabajó en el centro de investigación y consultoría organizacional CINCO de la Facultad de Minas de la Universidad Nacional de Colombia Sede Medellín, entidad que con recursos propios financió la investigación.

** Doctor en Ingeniería. Universidad Nacional de Colombia, MBA. Universidad De La Salle, Especialización en Mercadeo Gerencial. Universidad de Medellín, Ingeniero Civil. Profesor Asociado Universidad Nacional de Colombia Sede Medellín. Director Centro de Investigación y Consultoría Organizacional CINCO. Celular: 310840098 mdrojas@unal.edu.co

*** Magíster en Ingeniería Administrativa, Universidad Pontificia Bolivariana. Celular: 3217016228 1mmayaf@ unal.edu.co

**** MBA. Universidad de la Salle, Contadora pública. Universidad de Medellín. Profesora tiempo completo Fundación Universitaria Luis Amigó. Investigadora Grupo de investigación CONTAS de la Fundación Universitaria Luis Amigó. Facultad de Ciencias Administrativas, Económicas y Contables. Tel.: (574)4255309 maria.valenciaco@ amigo.edu.co 


\title{
Definition of a model for personal financial planning applied to the Colombian case
}

\begin{abstract}
Personal financial planning is a set of activities required for defining objectives, setting action plans, and satisfying financial needs of individuals. Different authors represent the personal financial planning process through models, indicating disadvantages to apply them in the Colombian environment since they have been created in developed countries; they suppose the existence of a financial planning industry and there are no academic studies which adapt them to meet the inherent needs of the environment. In order to solve such disadvantages, this research proposes the definition of a unifying personal financial planning model which embraces different elements that are identified from studied models, applicable in the Colombian environment.
\end{abstract}

Keywords: financial education; personal finances; personal financial planning.

\section{Definição de um modelo para o planejamento financeiro pessoal aplicado ao caso colombiano}

\begin{abstract}
Resumo
Planejamento financeiro pessoal é um conjunto de atividades requeridas para definir objetivos, estabelecer planos de ação e satisfazer necessidades financeiras de indivíduos. Diferentes autores representam o processo de planejamento financeiro pessoal por meio de modelos que indicam desvantagens para aplicá-los no contexto colombiano, porque foram criados em países desenvolvidos, além de supor a existência de uma indústria de planejamento financeiro e porque não existem estudos acadêmicos que os adaptem para as necessidades próprias desse entorno. Para solucionar as desvantagens, propõe-se, nesta pesquisa, a definição de um modelo unificador de planejamento financeiro pessoal que agrupe os diferentes elementos identificados a partir dos modelos estudados e que tenha aplicação no contexto colombiano.
\end{abstract}

Palavras-chave: educação financeira, finanças pessoais, planejamento financeiro pessoal. 


\section{INTRODUCCIÓN}

Las finanzas personales - $\mathrm{FP}-\mathrm{y}$ planeación financiera personal -PFP- son conceptos similares. FP es un conjunto de conceptos, herramientas y habilidades requeridas para solucionar problemas y tomar decisiones financieras personales; PFP es un concepto dinámico, que referencia a un conjunto de actividades requeridas para la definición de objetivos y establecimiento de planes de acción, satisfacción de las necesidades financieras presentes y futuras de un individuo o familia, o para el mejoramiento de las posibilidades financieras. Así las FP corresponden al marco de referencia conceptual requerido para ejecutar planeación financiera personal.

La PFP avanza en los últimos 25 a 30 años, especialmente en Estados Unidos, donde existe una industria de servicios financieros, asociaciones de profesionales, entidades que definen estándares para la aplicación de la PFP y la certificación de los profesionales en esta disciplina, para garantizar el bienestar del público en general. Para su aplicación se presentan diversos modelos que muestran el mecanismo de operación. Estos modelos presentan desventajas para aplicarlos en el ámbito colombiano porque son definidos en países desarrollados, suponiendo la existencia de una industria y una educación financiera suficiente.

Esta investigación propone un modelo de PFP con características definidas para el contexto colombiano, que permita tener un marco de referencia desarrollado en FP y fomentar la educación financiera. El modelo propuesto se construye con base en los modelos estudiados, cuya estructura sirve como marco para la construcción del mismo.

\section{MARCO TEÓRICO}

\subsection{Finanzas personales y planeación financiera personal}

En la tabla 1, se presentan los conceptos de FP, y en la tabla 2 los conceptos de PFP:

Tabla 1: Conceptos FP

\begin{tabular}{|l|l|}
\hline \multicolumn{1}{|c|}{ Autor } & \multicolumn{1}{c|}{ Definición } \\
\hline $\begin{array}{l}\text { Jacob, Hudson, } \\
\text { and Bush [1] }\end{array}$ & $\begin{array}{l}\text { Conceptos, habilidades, prácticas, reglas, normas e información para realizar acti- } \\
\text { vidades financieras. Financieras involucra un rango de actividades diarias asociadas } \\
\text { al manejo de fondos, tarjetas de crédito, seguros e inversiones. }\end{array}$ \\
\hline $\begin{array}{l}\text { Schuchardt et } \\
\text { al. [2] }\end{array}$ & $\begin{array}{l}\text { Profesión interdisciplinaria, donde se aplican conceptos: financieros, de gestión, } \\
\text { recursos, educación de los consumidores, sociología y psicología de toma de decisio- } \\
\text { nes para entender las formas como los individuos adquieren, desarrollan y asignan } \\
\text { recursos monetarios para sus necesidades financieras presentes y futuras. }\end{array}$ \\
\hline
\end{tabular}




\begin{tabular}{|l|l|}
\hline \multicolumn{1}{|c|}{ Autor } & \multicolumn{1}{c|}{ Definición } \\
\hline $\begin{array}{l}\text { Struwig and } \\
\text { Plaatjes [3] }\end{array}$ & $\begin{array}{l}\text { Conjunto de actividades, planear, toma de decisiones, organizar, y control de la asig- } \\
\text { nación de ingresos y acumulación de riqueza por un individuo, con el propósito de } \\
\text { alcanzar los objetivos financieros implícitos o explícitos de manera eficiente y eficaz. }\end{array}$ \\
\hline Remund [4] & Es la habilidad personal para entender y usar conceptos financieros \\
\hline Huston [5] & $\begin{array}{l}\text { Es la conciencia y el conocimiento de los instrumentos financieros y su aplicación en } \\
\text { los negocios y en la vida personal. }\end{array}$ \\
\hline Taft et al., [6] & $\begin{array}{l}\text { La habilidad de balancear una cuenta bancaria, preparar un presupuesto, ahorrar para } \\
\text { el futuro y aprender estrategias para administrar la deuda. }\end{array}$ \\
\hline
\end{tabular}

Fuente: elaboración propia, 2015

En las definiciones de FP se extraen elementos comunes: conocimientos, conceptos, habilidades, prácticas, reglas, normas, información, conjunto de actividades y conciencia, necesarios para solución de problemas financieros, manejo de productos financieros, adquisición, desarrollo y asignación de recursos monetarios para cubrir las necesidades financieras, toma de decisiones, y acumulación de riqueza. PFP es un conjunto de conceptos requeridos para tomar decisiones financieras. Ver tabla 2.

Tabla 2: Definiciones planeación financiera personal

\begin{tabular}{|l|l|}
\hline \multicolumn{1}{|c|}{ Autor } & \multicolumn{1}{c|}{ Definición } \\
\hline (CFP, n.d.) [7] & $\begin{array}{l}\text { Proceso que determina cómo el individuo cumple los objetivos de vida a través de la } \\
\text { adecuada gestión de los recursos financieros. }\end{array}$ \\
\hline Swart [8] & $\begin{array}{l}\text { Proceso de gestión del hogar individual que incluye la planificación, organización, di- } \\
\text { rección y control de los asuntos financieros de la familia. }\end{array}$ \\
\hline $\begin{array}{l}\text { Ruiz and } \\
\text { Bergés [9] }\end{array}$ & $\begin{array}{l}\text { Conjunto de decisiones realizadas a lo largo del ciclo vital de una unidad familiar para } \\
\text { de dar respuesta a sus necesidades financieras presentes y futuras. }\end{array}$ \\
\hline $\begin{array}{l}\text { Cooper and } \\
\text { Worsham [10] }\end{array}$ & $\begin{array}{l}\text { Proceso que busca determinar los objetivos financieros de los clientes y proporcionar un } \\
\text { plan para alcanzar los objetivos. }\end{array}$ \\
\hline Altfest [11] & $\begin{array}{l}\text { Método de preparación familiar para afrontar las futuras necesidades financieras de } \\
\text { forma eficiente. }\end{array}$ \\
\hline $\begin{array}{l}\text { Gitman and } \\
\text { Joehnk [12] }\end{array}$ & $\begin{array}{l}\text { Proceso que ayuda a las personas a definir los objetivos financieros y a desarrollar las } \\
\text { estrategias apropiadas para alcanzarlos. }\end{array}$ \\
\hline $\begin{array}{l}\text { Warschauer } \\
\text { [13] }\end{array}$ & $\begin{array}{l}\text { Proceso que tiene en cuenta la personalidad del cliente, posición financiera, medio ambi- } \\
\text { ente socio económico y jurídico, para la adopción de estrategias y el uso de herramientas } \\
\text { financieras para la consecución de los objetivos financieros del cliente. }\end{array}$ \\
\hline Gehring [14] & Profesión que ayuda a las personas a tomar buenas decisiones. \\
\hline
\end{tabular}


PFP es un conjunto de actividades para definir objetivos y establecer planes de acción que permiten satisfacer necesidades financieras presentes y futuras del individuo. FP refiere al marco requerido en planeación financiera personal como proceso dinámico.

\section{MODELOS DE PLANEACIÓN FINANCIERA EXISTENTES}

El objetivo es identificar, generalizar y esquematizar los componentes.

\subsection{Model of Certified Financial Planner Board of Standards -CFP Board-}

La figura 1 muestra tres aspectos importantes del CFP Board: (1) Análisis de la situación personal actual. (2) Definición de objetivos. (3) Medición durante el proceso:

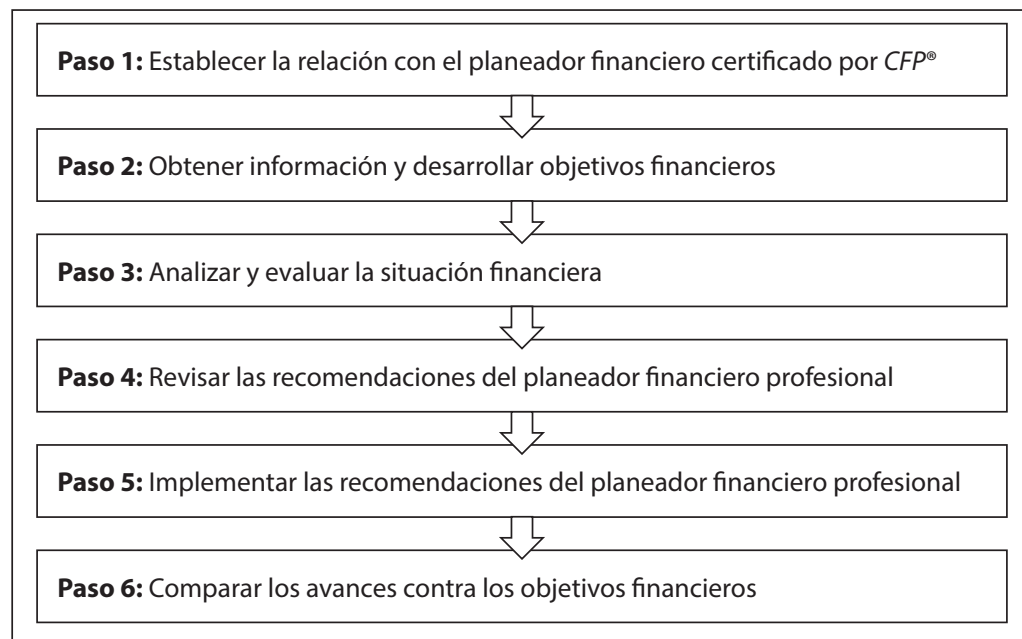

Figura 1. Modelo CFP Board

Fuente: elaboración propia, 2015

\subsection{Modelo de Chieffe y Rakes [15]}

En la tabla 3, se observa que es creado para aplicar por cualquier persona. El modelo integra los elementos que son cubiertos en la planeación financiera y las FP.

Tabla 3. Modelo Chieffe y Rakes [15]

\begin{tabular}{|c|c|c|}
\hline & Presente & Futuro \\
\hline $\begin{array}{l}\text { Eventos } \\
\text { finan- } \\
\text { cieros } \\
\text { previstos }\end{array}$ & $\begin{array}{l}\text { Administración del dinero } \\
\text { Presupuesto: Ingresos, Gastos de manutención, } \\
\text { Ahorros, Créditos } \\
\text { Planeación tributaria: Deducciones, Impuestos }\end{array}$ & $\begin{array}{l}\text { Invirtiendo por lo objetivos } \\
\text { Planeación de las inversiones: Acciones y Bo- } \\
\text { nos, Fondos mutuos, Propiedad raíz } \\
\text { Planeación de la jubilación: Fondo de pensio- } \\
\text { nes, Pensiones voluntarias }\end{array}$ \\
\hline
\end{tabular}




\begin{tabular}{|c|c|c|}
\hline & Presente & Futuro \\
\hline $\begin{array}{l}\text { Eventos } \\
\text { financie- } \\
\text { ros no } \\
\text { previstos }\end{array}$ & $\begin{array}{l}\text { Planeación de emergencias } \\
\text { Administración del riesgo: Fondo de emergen- } \\
\text { cias, Líneas de Crédito } \\
\text { Seguros: Propiedad, Salud, Deuda. }\end{array}$ & $\begin{array}{l}\text { Planeación de la transferencia } \\
\text { Planeación de la sucesión: Testamentos, Pla- } \\
\text { neación fiscal, Fideicomisos, Seguros, Acuer- } \\
\text { dos comerciales, Planes de caridad }\end{array}$ \\
\hline
\end{tabular}

\subsection{Modelo de Ruiz y Bergés [9]}

La figura 2 representa el proceso de planificación definido por Ruiz y Bergés, y comprende una serie de etapas que interactúan entre sí para gestionar de forma ordenada y dinámica en el tiempo la gestión integral de las finanzas familiares.

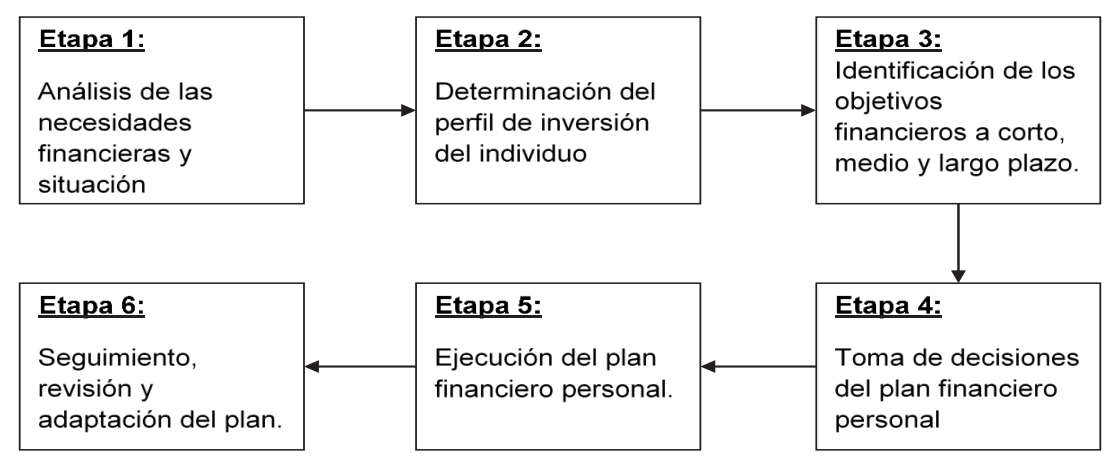

Figura 2: Modelo Gestión Integral de las finanzas familiares de Ruiz and Bergés Fuente: [9]

\subsection{Modelo de Cooper y Worsham [10]}

Cooper y Worsham definen la PF como el proceso que busca determinar los objetivos financieros del cliente y proporcionar un plan para alcanzarlos, el proceso de planeación financiero consta de 6 pasos como se ilustra en la figura 3.

\subsection{Modelo de Gehring [14]}

Gehring define la planeación financiera como profesión que ayuda a las personas a tomar decisiones financieras pertinentes. El proceso de planeación financiera se describe mediante un modelo de decisión conformado por cuatro factores que interactúan entre sí y considerados todos en conjunto, el cual es representado en la figura 4. 


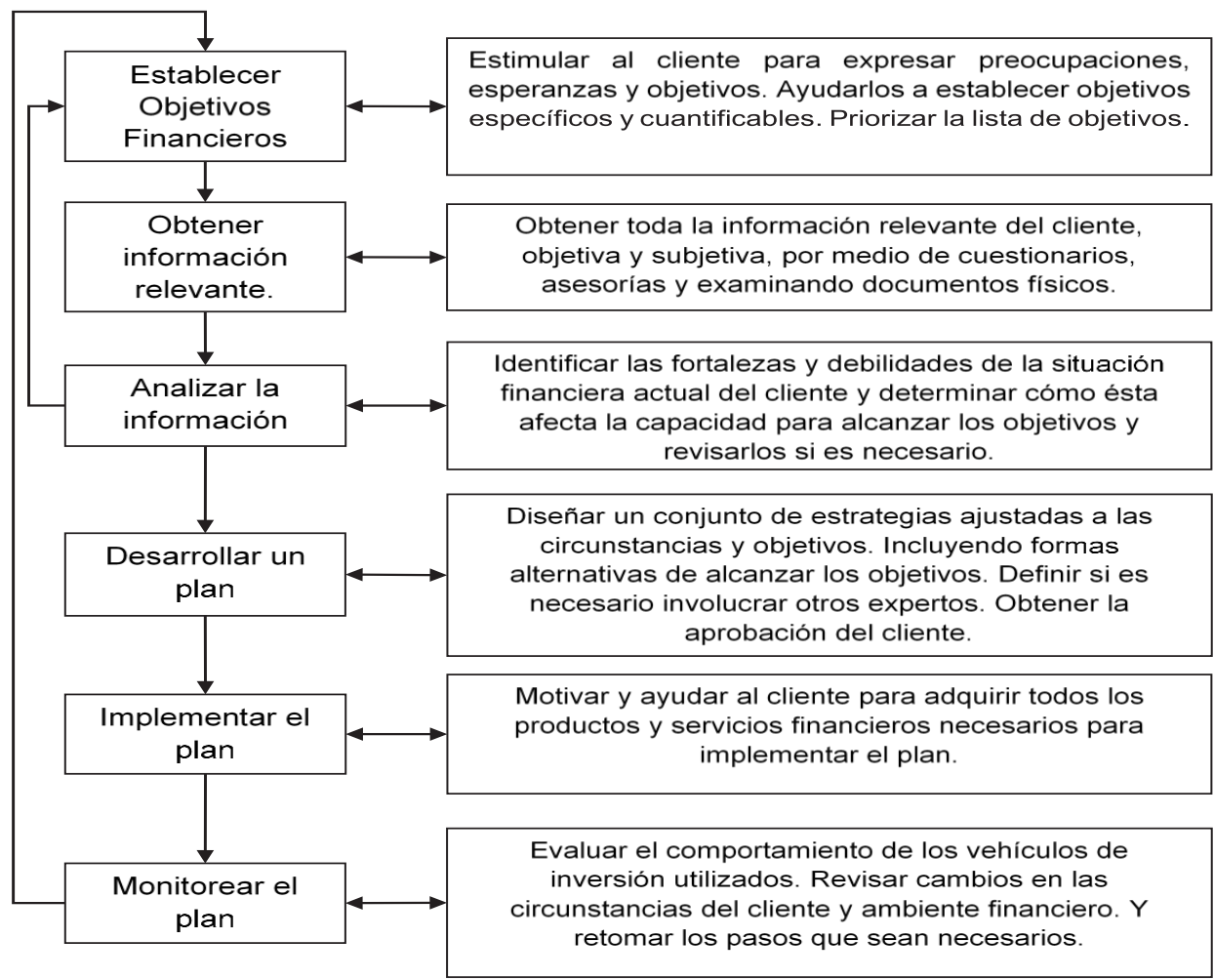

Figura 3: El proceso de planeación financiera Cooper and Worsham

Fuente: [10]

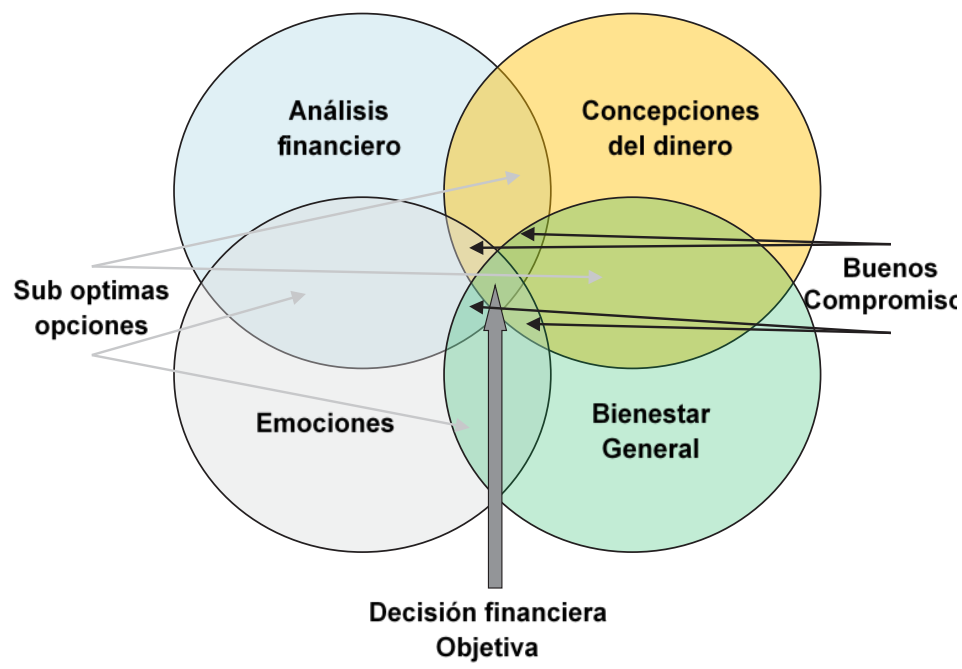

Figura 4: El proceso de planeación financiera Gehring

Fuente: [14] 


\subsection{Modelo Nissenbaum, Raasch and Ratner [16]}

En la figura 5 se muestra el proceso que permite a la persona hacerse cargo de las finanzas. El primero describe la necesidad de identificar y medir la posición financiera actual de la persona, a partir del análisis del patrimonio neto y del flujo de caja, siendo el punto inicial para la medición del progreso hacia la consecución de los objetivos.

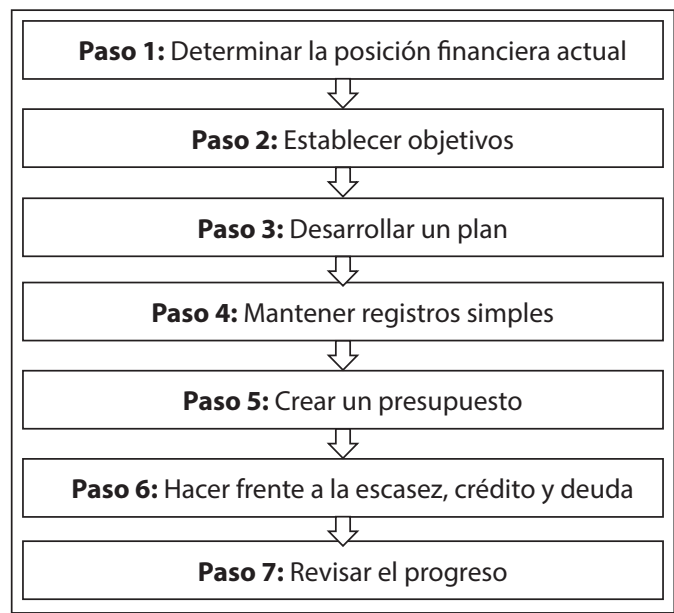

Figura 5: El proceso de planeación financiera Nissenbaum, Raasch, and Ratner Fuente: [16].

\section{ANÁLISIS DE LOS MODELOS DE PLANEACIÓN FINANCIERA DEFINIDOS}

Analizando y comparando los modelos, se construyó la matriz (ver tabla 4), donde se muestran los elementos expuestos por cada uno, y se identifican componentes del modelo PFP y los vacíos. Luego, los componentes se integran al modelo unificado, y los vacíos se emplearán para la conformación del modelo que supera esas deficiencias.

Tabla 4: Análisis comparativo modelos descritos.

\begin{tabular}{|l|c|c|c|c|c|c|}
\cline { 2 - 7 } \multicolumn{1}{c|}{} & \multicolumn{9}{c|}{ Concepto } & $\begin{array}{c}\text { CFP } \\
\text { Board } \\
(\text { n.d.) } \\
{[7]}\end{array}$ & $\begin{array}{c}\text { Chieffe y } \\
\text { Rakes } \\
{[15]}\end{array}$ & $\begin{array}{c}\text { Ruiz y } \\
\text { Bergés } \\
{[9]}\end{array}$ & $\begin{array}{c}\text { Cooper y } \\
\text { Worsham } \\
{[10]}\end{array}$ & $\begin{array}{c}\text { Nissenbaum, } \\
\text { Raasch, and } \\
\text { Ratner [16] }\end{array}$ & $\begin{array}{c}\text { Gehring } \\
{[14]}\end{array}$ \\
\hline Relación planeador financiero certificado & $\mathrm{X}$ & & & & & \\
\hline Establecimiento de objetivos financieros & $\mathrm{X}$ & $\mathrm{X}$ & $\mathrm{X}$ & $\mathrm{X}$ & $\mathrm{X}$ & \\
\hline Evaluación situación financiera & $\mathrm{X}$ & & $\mathrm{X}$ & $\mathrm{X}$ & $\mathrm{X}$ & $\mathrm{X}$ \\
\hline Establecimiento plan financiero & $\mathrm{X}$ & $\mathrm{X}$ & $\mathrm{X}$ & $\mathrm{X}$ & $\mathrm{X}$ & \\
\hline
\end{tabular}




\begin{tabular}{|l|c|c|c|c|c|c|}
\cline { 2 - 8 } \multicolumn{1}{c|}{} & \multicolumn{7}{c|}{ Concepto } & $\begin{array}{c}\text { CFP } \\
\text { Board } \\
\text { (n.d.) } \\
{[7]}\end{array}$ & $\begin{array}{c}\text { Chieffe y } \\
\text { Rakes } \\
{[15]}\end{array}$ & $\begin{array}{c}\text { Ruiz y } \\
\text { Bergés } \\
{[9]}\end{array}$ & $\begin{array}{c}\text { Cooper y } \\
\text { Worsham } \\
{[10]}\end{array}$ & $\begin{array}{c}\text { Nissenbaum, } \\
\text { Raasch, and } \\
\text { Ratner [16] }\end{array}$ & $\begin{array}{c}\text { Gehring } \\
{[14]}\end{array}$ \\
\hline Implementación plan financiero & $\mathrm{X}$ & $\mathrm{X}$ & $\mathrm{X}$ & $\mathrm{X}$ & & $\mathrm{X}$ \\
\hline Validación / Monitoreo del plan financiero & $\mathrm{X}$ & $\mathrm{X}$ & $\mathrm{X}$ & $\mathrm{X}$ & $\mathrm{X}$ & \\
\hline Naturaleza de los eventos financieros & & $\mathrm{X}$ & & & $\mathrm{X}$ & \\
\hline Horizonte temporal & & $\mathrm{X}$ & $\mathrm{X}$ & & $\mathrm{X}$ & \\
\hline Perfil de riesgo de la persona. & & & $\mathrm{X}$ & & & $\mathrm{X}$ \\
\hline Concepciones del dinero & & & & & & $\mathrm{X}$ \\
\hline Emociones & & & & & & $\mathrm{X}$ \\
\hline Bienestar General & & & & & & \\
\hline Registro numérico del evento. & & & & & \\
\hline
\end{tabular}

Fuente: elaboración propia, 2015

El marco de referencia incluye un conjunto de habilidades y conocimientos necesarios para el ejercicio de la PFP (Financial Planning Standards Board, [7]). En la tabla 5 se muestran las áreas de conocimiento y se clasifican en ocho categorías.

Tabla 5. Matriz de componentes de la planeación financiera personal.

\begin{tabular}{|c|c|c|c|c|c|c|c|c|c|}
\hline $\begin{array}{c}\text { Autores } \\
\text { Componentes }\end{array}$ & $\begin{array}{l}F P S B \\
(2011)\end{array}$ & $\begin{array}{l}\text { L. J. Gitman, } \\
\text { Joehnk, \& } \\
\text { Billingsley, } \\
\text { (2014) }\end{array}$ & $\begin{array}{l}\text { Garman \& } \\
\text { Forgues, } \\
\text { (2012) }\end{array}$ & $\begin{array}{c}\text { Struwig \& } \\
\text { Plaatjes } \\
\text { (2007) }\end{array}$ & \begin{tabular}{|c|} 
Nissenbaum, \\
Raasch, Ratner, \\
\& Ernst\&young \\
(2004)
\end{tabular} & $\begin{array}{l}\text { Swart } \\
(2004)\end{array}$ & $\begin{array}{l}\text { Woerheide } \\
\text { (2003) }\end{array}$ & $\begin{array}{c}\text { Cooper \& } \\
\text { Worsham } \\
\text { (2003) }\end{array}$ & $\begin{array}{l}\text { Rosefsky } \\
\text { (2001) }\end{array}$ \\
\hline $\begin{array}{l}\text { Conceptualiza- } \\
\text { ción Planeación } \\
\text { Financiera } \\
\text { Personal }\end{array}$ & $\begin{array}{l}\text { Principios y } \\
\text { práctica de } \\
\text { la planeación } \\
\text { financiera } \\
\text { Planeación } \\
\text { financiera } \\
\text { integrada. }\end{array}$ & $\begin{array}{l}\text { Fundamentos } \\
\text { de la planeación } \\
\text { financiera }\end{array}$ & $\begin{array}{l}\text { Planeación } \\
\text { financiera } \\
\text { personal. }\end{array}$ & $\begin{array}{l}\text { Conceptos } \\
\text { básicos de } \\
\text { FP. } \\
\text { Adminis- } \\
\text { tración de } \\
\text { las FP. }\end{array}$ & $\begin{array}{l}\text { Proceso de } \\
\text { planeación } \\
\text { financiera } \\
\text { personal }\end{array}$ & $\begin{array}{l}\text { Planeación } \\
\text { Financiera. }\end{array}$ & $\begin{array}{l}\text { Planeación } \\
\text { financiera. }\end{array}$ & $\begin{array}{l}\text { Principios } \\
\text { generales } \\
\text { de la } \\
\text { planeación } \\
\text { financiera. }\end{array}$ & $\begin{array}{l}\text { Economía } \\
\text { Familiar }\end{array}$ \\
\hline $\begin{array}{l}\text { Gestión de } \\
\text { Activos e } \\
\text { Inversiones }\end{array}$ & $\begin{array}{l}\text { Gestión } \\
\text { Financiera. } \\
\text { Gestión de } \\
\text { Activos. }\end{array}$ & $\begin{array}{l}\text { Gestión de los } \\
\text { bienes básicos. } \\
\text { Gestión de } \\
\text { inversiones. }\end{array}$ & Inversiones & $\begin{array}{l}\text { Inversión } \\
\text { en recursos } \\
\text { financieros. }\end{array}$ & $\begin{array}{l}\text { Gestión de } \\
\text { los vehículos } \\
\text { de inversión, } \\
\text { y mercados } \\
\text { financieros. }\end{array}$ & $\begin{array}{l}\text { Planea- } \\
\text { ción de } \\
\text { inversiones. }\end{array}$ & $\begin{array}{l}\text { Inversión de } \\
\text { los recursos } \\
\text { financieros. }\end{array}$ & $\begin{array}{l}\text { Planeación } \\
\text { de las } \\
\text { inversiones. }\end{array}$ & $\begin{array}{l}\text { Inversión de } \\
\text { los recursos }\end{array}$ \\
\hline $\begin{array}{l}\text { Gestión del } \\
\text { Endeudamiento }\end{array}$ & $\begin{array}{l}\text { Gestión } \\
\text { Financiera. }\end{array}$ & $\begin{array}{l}\text { Gestión del } \\
\text { crédito. }\end{array}$ & $\begin{array}{l}\text { Adminis- } \\
\text { tración del } \\
\text { efectivo }\end{array}$ & & & $\begin{array}{l}\text { Planeación } \\
\text { del endeu- } \\
\text { damiento. }\end{array}$ & Vivienda. & & $\begin{array}{l}\text { Obtención de } \\
\text { recursos. } \\
\text { Financiación } \\
\text { de vivienda. }\end{array}$ \\
\hline
\end{tabular}


164 M. D. Rojas López - L. M. Maya Fernández - M. E. Valencia

\begin{tabular}{|c|c|c|c|c|c|c|c|c|c|}
\hline $\begin{array}{c}\text { Autores } \\
\text { Componentes }\end{array}$ & $\begin{array}{l}\text { FPSB } \\
(2011)\end{array}$ & $\begin{array}{l}\text { L. J. Gitman, } \\
\text { Joehnk, \& } \\
\text { Billingsley, } \\
\text { (2014) }\end{array}$ & $\begin{array}{l}\text { Garman \& } \\
\text { Forgues, } \\
\text { (2012) }\end{array}$ & $\begin{array}{c}\text { Struwig \& } \\
\text { Plaatjes } \\
\text { (2007) }\end{array}$ & $\begin{array}{c}\text { Nissenbaum, } \\
\text { Raasch, Ratner, } \\
\text { \& Ernst\&young } \\
\text { (2004) }\end{array}$ & $\begin{array}{l}\text { Swart } \\
(2004)\end{array}$ & $\begin{array}{c}\text { Woerheide } \\
\text { (2003) }\end{array}$ & $\begin{array}{c}\text { Cooper \& } \\
\text { Worsham } \\
\text { (2003) }\end{array}$ & $\begin{array}{c}\text { Rosefsky } \\
\text { (2001) }\end{array}$ \\
\hline $\begin{array}{l}\text { Gestión del } \\
\text { efectivo (Ingre- } \\
\text { sos y Gastos) }\end{array}$ & $\begin{array}{l}\text { Gestión } \\
\text { Financiera. }\end{array}$ & $\begin{array}{l}\text { Gestión de los } \\
\text { bienes básicos }\end{array}$ & $\begin{array}{l}\text { Adminis- } \\
\text { tración del } \\
\text { efectivo }\end{array}$ & & & & & & \\
\hline $\begin{array}{l}\text { Gestión de ries- } \\
\text { go y seguros }\end{array}$ & $\begin{array}{l}\text { Gestión del } \\
\text { riesgo. }\end{array}$ & $\begin{array}{l}\text { Gestión de las } \\
\text { necesidades de } \\
\text { seguros }\end{array}$ & $\begin{array}{l}\text { Protección } \\
\text { de los } \\
\text { ingresos y } \\
\text { activos }\end{array}$ & $\begin{array}{l}\text { Adminis- } \\
\text { tración del } \\
\text { riesgo }\end{array}$ & $\begin{array}{l}\text { Protección de } \\
\text { la familia y los } \\
\text { activos. }\end{array}$ & $\begin{array}{l}\text { Planea- } \\
\text { ción de la } \\
\text { protección }\end{array}$ & $\begin{array}{l}\text { Protección } \\
\text { de los } \\
\text { recursos }\end{array}$ & $\begin{array}{l}\text { Planeación } \\
\text { de seguros y } \\
\text { adminis- } \\
\text { tración del } \\
\text { riesgo }\end{array}$ & $\begin{array}{l}\text { Protección de } \\
\text { los recursos }\end{array}$ \\
\hline $\begin{array}{l}\text { Gestión } \\
\text { tributaria }\end{array}$ & $\begin{array}{l}\text { Planeación } \\
\text { tributaria }\end{array}$ & $\begin{array}{l}\text { Gestión de } \\
\text { Impuestos }\end{array}$ & $\begin{array}{l}\text { Adminis- } \\
\text { tración del } \\
\text { efectivo }\end{array}$ & & $\begin{array}{l}\text { Planeación } \\
\text { tributaria y } \\
\text { deducciones }\end{array}$ & $\begin{array}{l}\text { Planea- } \\
\text { ción del } \\
\text { impuesto a } \\
\text { la renta }\end{array}$ & & $\begin{array}{l}\text { Planeación } \\
\text { del impues- } \\
\text { to de renta }\end{array}$ & \\
\hline $\begin{array}{l}\text { Gestión de } \\
\text { Jubilación y } \\
\text { sucesión }\end{array}$ & $\begin{array}{l}\text { Planeación } \\
\text { del retiro / } \\
\text { jubilación } \\
\text { Planeación de } \\
\text { la sucesión }\end{array}$ & $\begin{array}{l}\text { Planeación de } \\
\text { la jubilación y } \\
\text { sucesión }\end{array}$ & $\begin{array}{l}\text { Planea- } \\
\text { ción de la } \\
\text { jubilación y } \\
\text { sucesión }\end{array}$ & $\begin{array}{l}\text { Planifica- } \\
\text { ción del } \\
\text { futuro }\end{array}$ & $\begin{array}{l}\text { Planeación de } \\
\text { la sucesión. }\end{array}$ & $\begin{array}{l}\text { Planea- } \\
\text { ción de la } \\
\text { jubilación } \\
\text { Planea- } \\
\text { ción de la } \\
\text { sucesión } \\
\text { herencia }\end{array}$ & $\begin{array}{l}\text { Planeación } \\
\text { del retiro } \\
\text { Planea- } \\
\text { ción de la } \\
\text { sucesión o } \\
\text { herencia }\end{array}$ & $\begin{array}{l}\text { Planeación } \\
\text { de la } \\
\text { jubilación } \\
\text { Planea- } \\
\text { ción de la } \\
\text { sucesión/ } \\
\text { Herencia }\end{array}$ & \\
\hline $\begin{array}{l}\text { Componentes } \\
\text { no clasificados }\end{array}$ & & & & Diversos & & $\begin{array}{l}\text { Planeación } \\
\text { de: la salud, } \\
\text { Emigración } \\
\text { Profesión }\end{array}$ & & $\begin{array}{l}\text { Planeación } \\
\text { de los be- } \\
\text { neficios del } \\
\text { empleado }\end{array}$ & \\
\hline
\end{tabular}

Fuente: elaboración propia, 2015

La PFP tiene avances en los últimos 25 a 30 años, especialmente en USA, donde existe la industria de servicios de asesoramiento financiero, asociaciones de profesionales, entidades que definen estándares para aplicar la PFP y la certificación de los profesionales de este campo para garantizar el bienestar del público en general.

Es necesario profundizar en aspectos como (1) Establecimiento de una teoría propia facilitando futuras investigaciones. (2) Mejoramiento de la formación en FP usando técnicas sofisticadas. (3) Desarrollando libros de texto con calidad. (4) Estableciendo nuevos centros de formación en planeación financiera. (5) Incorporando aspectos del comportamiento humano en la formación en FP, [11] es decir, es necesario desarrollar un modelo de decisión completo y robusto [14].

Para desarrollar las FP es necesario mayor involucramiento de la academia [11]. Las universidades tienen un rol importante en el desarrollo de la PFP como profesión, y en la formación de profesionales [21].

La investigación presenta un modelo de PFP aplicable al contexto colombiano, incorporando características del mercado financiero nacional que sirva de soporte para la planeación financiera personal en Colombia. 


\section{MODELO PARA LA PFP APLICADO AL CASO COLOMBIANO}

La figura 6, integra los elementos identificados en los modelos existentes y se complementan generando un modelo integrador.

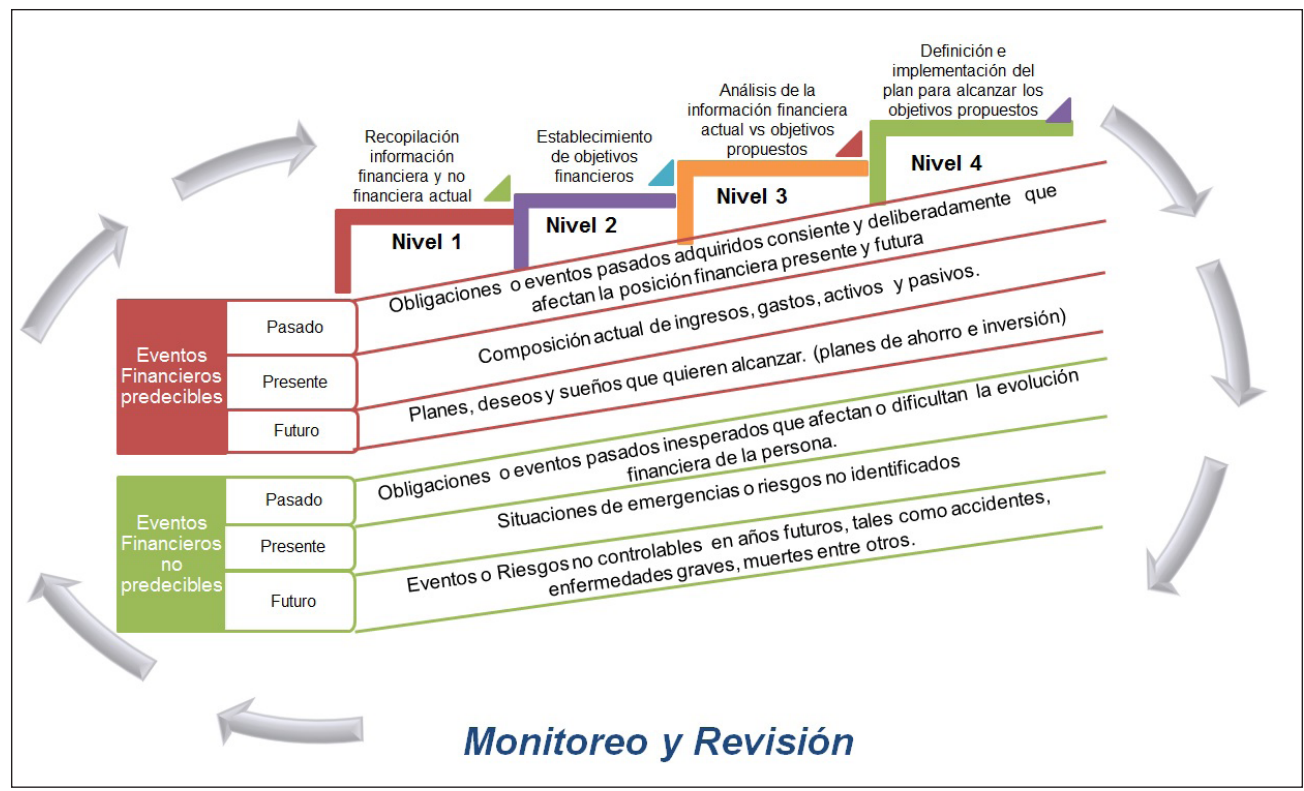

Figura 6: Modelo para la PFP aplicado al caso colombiano

Fuente: Elaboración propia, 2015

Componente 1 - Naturaleza de los eventos: Se reconoce la naturaleza de los eventos que afectan las FP, y se analizan las estrategias a diseñar durante la planeación. Se establecen dos tipos de eventos: la capacidad de la ocurrencia y el impacto del mismo.

- Evento financiero predecible: suceso que es fácil de identificar, fecha de ocurrencia y el impacto. El origen se sitúa en compromisos adquiridos en el pasado.

- Evento financiero no predecible: suceso que no se puede prever la fecha de ocurrencia y difícil estimar el impacto en la posición financiera.

Componente 2 - Tiempo: refiere a la temporalidad del momento en el que tienen lugar los eventos y el tiempo. El modelo propuesto establece 3 dimensiones de tiempo:

- Pasado: eventos en una fecha anterior que afectan la posición financiera presente o futura, generando impactos positivos o negativos, representan obligaciones previas. 
- Presente: posición actual, eventos presentes generadores de ingresos, egresos, obligaciones, derechos, proyectos, entre otras.

- Futuro: horizonte de tiempo de la planeación y revisión. Identificación de eventos en el futuro que afectarán PFP, incluye: descripción de planes, deseos y sueños.

Componente 3 - Monitorización y revisión: Es la sistemática documentación de resultados del proceso, evaluando si el plan se ejecuta según lo previsto. [22], medir el desempeño y tener un sistema de alertas tempranas para las acciones correctivas [23].

Se requiere por dos razones; primera, el modelo es aplicado donde la realidad cambia, implica que el modelo requiere adaptación. La segunda, la correlación entre actividades descritas, implicando que un hallazgo obliga a ajustar actividades precedentes.

Componente 4 - Niveles del proceso: Es una serie de actividades lógicas y relevantes necesarias para consolidar un proceso dinámico, así:

Nivel 1 - Recopilación de información financiera y no financiera: Información relevante del individuo para conocer los elementos del pasado y el presente.

La información es financiera y no financiera, para reconocer aspectos cuantitativos y cualitativos. En la financiera: flujo de ingresos y gastos, flujos de ahorros e inversión, deudas y obligaciones con terceros, seguros contratados. En la no financiera: gustos y preferencias, paradigmas sobre las finanzas, caracterización del entorno, identificación de la salud física, mental y emocional y evaluación de la exposición al riesgo

En este nivel se define en qué lugar del ciclo de vida se ubica, es decir, si está iniciando o está en nivel medio o se ubica en fase final. Las personas se clasifican en los siguientes grupos:

- Principiantes: personas vinculadas por primera vez al mercado laboral, normalmente ingresos bajos para manutención. Emprenden proyectos como vivienda, educación y viajes. El horizonte de inversión es largo plazo.

- Experimentados: personas alrededor de la mitad de la vida laboral, ingresos en buen nivel, ejecutando inversión y aumento del patrimonio. El horizonte de inversión es mediano a largo plazo. En esta etapa la planeación tributaria es una necesidad.

- Maduros: se ubican en los últimos años del ciclo productivo, donde la mayor necesidad es garantizar una adecuada jubilación, para lo cual ajustan la PF al logro de esta. En este caso, el horizonte de inversión es corto. 
Nivel 2 - Establecimiento de objetivos financieros: El desarrollo de planes de acción requiere tiempo [24]. Los modelos no son excluyentes, son combinables, permitiendo adaptarlos en la definición de objetivos. Locke, Latham, Smith, Wood, and Bandura [17] establecen que definir objetivos requiere cinco principios (ver tabla 6):

Tabla 6: Cinco principios de la fijación de objetivos

\begin{tabular}{|c|c|c|c|c|}
\hline \multicolumn{5}{|c|}{ Principios } \\
\hline Claridad & Retadores & Compromiso & Retroalimentación & $\begin{array}{c}\text { Complejidad de la } \\
\text { tarea }\end{array}$ \\
\hline $\begin{array}{l}\text { Que sean } \\
\text { específicos } \\
\text { y sin } \\
\text { ambigüedades }\end{array}$ & $\begin{array}{l}\text { Poseer el nivel correcto } \\
\text { de desafío, ni elevado } \\
\text { ni bajo porque ambos } \\
\text { extremos generan } \\
\text { desmotivación }\end{array}$ & $\begin{array}{l}\text { Requieren ser } \\
\text { entendidos } \\
\text { y poseer el } \\
\text { compromiso } \\
\text { de las personas } \\
\text { involucradas. }\end{array}$ & $\begin{array}{l}\text { A la mayoría de } \\
\text { las personas les } \\
\text { gusta saber cómo } \\
\text { está siendo el } \\
\text { desempeño versus } \\
\text { lo que se espera de } \\
\text { ellos }\end{array}$ & $\begin{array}{l}\text { Simplificar la } \\
\text { definición de } \\
\text { objetivos, por } \\
\text { medio de dividir } \\
\text { procesos por } \\
\text { áreas con tiempo } \\
\text { suficiente. }\end{array}$ \\
\hline
\end{tabular}

Fuente: elaboración propia, 2015

Yemm [21] define la planeación como SMARTER: tiene origen en el modelo SMART inventado por Dorna [22], estableciendo que garantizar el éxito requiere ser:

- Específicos (“S for Specific”), identificar claramente la acción involucrada.

- Medibles ("M for Measurable"), es indispensable tener una medida que permita cuantificar el resultado. Cuando el objetivo es cuantitativo es fácil definir el indicador.

- Alcanzable o acordado. ("A for Achievable or Agreed"): Los objetivos deben ser retadores y ambiciosos. Que se logre en los tiempos establecidos.

- Relevante o realista ("R for Relevant or Realistic"): establecer si el objetivo es realmente importante, cómo contribuye a la consecución de los demás.

- Delimitados en el tiempo ("T for Time - Bound"): delimitar fecha para el logro del objetivo, establecer prioridades y promover acciones.

- Evaluación ("E for Evaluated"): se definen acciones que permitan evaluar el desarrollo y progreso, poder tomar las acciones correctivas oportunamente.

- Revisión / recompensa ("R for Reviewed or Rewarded"): Hace referencia a la revisión del desempeño en la consecución de un objetivo. 
Definir objetivos es factor clave de éxito; es imperativo que sean: (1) Específicos para reducir la complejidad. (2) Medibles: para que cuantitativamente se valore el progreso hacia la consecución del resultado. (3) Alcanzables: un reto para la persona que, a su vez, estén acordes con la actualidad financiera y los recursos. (4) Delimitados en el tiempo.

Nivel 3 - Análisis de la información financiera actual versus objetivos propuestos

El objetivo es identificar brechas entre lo actual y los objetivos. El análisis de lo actual se orienta por medio del patrimonio neto, flujo de caja y análisis combinado de ambos.

El cálculo del patrimonio neto es la resta entre activos (recursos) y pasivos (deudas). Permite analizar tres áreas, así: (1) Identificar y valorar los recursos propiedad de la persona, tipo de activo, si generan renta o no, si son de larga o corta duración. (2) Establecer nivel de endeudamiento actual, tiempo, costo y origen. (3) Cuantificar la posición financiera neta que indica el nivel de riqueza propia.

El flujo de caja identifica el nivel de ingresos y gastos, con una periodicidad mínima mensual, estableciendo si el nivel de ingresos es suficiente o no. El dimensionamiento del flujo de gastos es necesario hacerlo realista y detallado.

Si el flujo de caja es negativo, se hace análisis cruzado entre el nivel de endeudamiento y gastos; este resultado se cubre con un mayor endeudamiento.

\section{Nivel 4 -Definición e implementación del plan financiero}

Se define e implementa el plan de acción para cada objetivo. Para la estructuración del plan financiero se crea una ficha donde: (1) Enuncie las acciones requeridas para llegar a la meta. (2) Establezca fechas guía para revisar el progreso. (3) En los casos que aplique, enuncie áreas que beneficien el desarrollo de la acción o afecten su ejecución. (4) Describa los productos financieros a utilizar.

Se elabora el presupuesto financiero donde se proyecten flujos de ingresos, gastos, deudas e inversiones para cada uno de los objetivos definidos. El plan financiero se consolida por medio de su implementación, que consiste en ejecutar las acciones definidas en los planes descritos para cada objetivo establecido.

\section{CONCLUSIONES}

En Colombia no hay evidencia sobre el tema de FP; en contraste se encuentra que en el ámbito internacional existen numerosas publicaciones sobre el objeto de estudio, permitiendo desarrollar y contextualizar esta disciplina. 
Asimismo, hay ausencia de programas académicos orientados a la educación financiera, en Educación Básica y Superior; evidencia de ello es el resultado en el 2014 de las pruebas internacionales de educación del Programa de Evaluación Internacional de Estudiantes (PISA), que realiza la Organización para la Cooperación y el Desarrollo Económico (OCDE) para evaluar distintas áreas académicas en diferentes países, donde en educación financiera los estudiantes de colegio de Colombia obtuvieron 379 puntos, y se ubicaron en el último lugar de los países evaluados.

A partir de iniciativas oficiales, como la creación del programa de inversión denominado el banco de oportunidades para acercar los servicios financieros a las personas de menores recursos, la ley de protección del consumidor financiero que establece la responsabilidad del sistema financiero en educación a los consumidores con respecto a productos y servicios y finalmente la iniciativa del Ministerio de Educación Nacional -MEN- se incluirán programas de desarrollo de competencias básicas en economía y finanzas.

A partir del análisis de los modelos presentados se concluye que un modelo unificador mínimamente contiene: definición de objetivos, evaluación de la situación financiera, definición e implementación de un plan y evaluación y monitorización del proceso.

El modelo de PFP propuesto logra los objetivos planteados porque cumple con la integración de los elementos identificados en el estudio de los modelos referenciados. La puesta en práctica del modelo de PFP requiere de un marco de referencia que representa el conjunto de habilidades que fundamentan la ejecución del plan financiero que se defina. Se agruparon y sintetizaron los conceptos en siete áreas de conocimiento, como conceptualización de la PFP, gestión de activos e inversiones, del endeudamiento, del efectivo, del riesgo, tributaria y gestión de la jubilación y sucesión.

\section{REFERENCIAS}

[1] Jacob, K., Hudson, S., and Bush, M. Tool for survival: An analysis of financial literacy programs for lowe - income families. Chicago: Woodstock Institute, 2000.

[2] Schuchardt, J., et al., Personal Finance : An Interdisciplinary Profession. Financial Counceling, 2007

[3] Struwig, F., and Plaatjes, W. Developing a framework to investigate the personal financial management knowledge if individuals. South African Jounal of Economic \& Management Sciences, 10(1), 21-32, 2007. 
[4] Remund, D. L. Financial Literacy Explicated: The Case for a Clearer Definition in an Increasingly Complex Economy. Journal of Consumer Affairs, 44(2), 276-295. doi:10.1111/j.17456606.2010.01169.x, 2010.

[5] Huston, S. J. Measuring Financial Literacy. Journal of Consumer Affairs, 44(2), 296-316. doi:10.1111/j.1745-6606.2010.01170.x, 2010.

[6] Taft, M. K., Hosein, Z. Z., and Mehrizi, S. M. T. The Relation between Financial Literacy, Financial Wellbeing and Financial Concerns. International Journal of Business and Management, 8(11), 63-75. doi:10.5539/ijbm.vol. 8, n. ${ }^{\circ} 1$,p. 63, 2013.

[7] FP, B. (n.d.). CFB Board. Retrieved from http://www.letsmakeaplan.org/learning-center/ FinancialPlanningProcess.

[8] Swart, N. Personal Financial Management (2nd ed., p. 432). Cape Town: Juta Academic, 2004.

[9] Ruiz, A., and Bergés, Á. El nuevo paradigma de las FP. En Portada-Bolsa de Madrid, 10-17, 2002 .

[10] Cooper, R. W., and Worsham, C. B. The Financial Planning Process. In R. Cooper and C. Worsham (Eds.), Foundations of Financial planning: An Overview (pp. 1-46). America College, 2003

[11] Altfest, L. Personal Financial Planning: Origins, Developments and a plan for Future Direction. The American Economist, 48(2), 53-60, 2004

[12] Gitman, L., and Joehnk, M. Personal Financial Planning. (A. Von \& S. Smart, Eds.) (Thomson So., pp. 2-30). Mason: Thomson Higher Education, 2008.

[13] Warschauer, T. Course Syllabus and readings. Financial Planning Practicum, 2010

[14] Gehring, N. Defining Financial Planning with the 4 factor decision model. Journal of Financial Planning, January, 19-21, 2013.

[15] Chieffe, N., Rakes, G. An integrated model for financial planning. Financial Services Review 8, 261-268, 1999

[16] Nissenbaum, M., Raasch, B., and Ratner, C. Ernst and Young's Personal Financial Planning Guide (5th ed., p. 552). John Wiley and Sons, Inc., 2004

[17] Locke, E., Latham, G. P., Smith, K. J., Wood, R. E., and Bandura, A. A theory of Goal Setting \& Task Performance. (Illustrate., p. 413). Englewood Cliffs, NJ, US: Prentice Hall, 1990.

[18] Gitman, L. J., Joehnk, M. D., and Billingsley, R. S. Personal Financial Planning (13th ed., p. 622). Mason: South-Western, Cengage Learning, 2014.

[19] Garman, T., \& Forgues, R. E. Personal Finance. (M. Reynolds, J. Chase, \& S. Fidler, Eds.) (11th ed., p. 559). Mason: South-Western Cengage Learning, 2012 
[20] Rosefsky, R. S. Personal Finance (p. 704). Hoboken, New Jersey: John Wiley \& Sons, Inc., 2001.

[21] Yemm, G. Financial Times Essential Guide to Leading Your Team: How to Set Goals, Measure Performance and Reward Talent (p. 184). FT Publishing: Pearson Education, 2012.

[22] Dorna, G. There's a S.M.A.R.T. way to write management's goals and objectives. Management Review (AMA FORUM), 70(11), 35-36, 1981. 